\title{
Recent dermatophyte divergence revealed by comparative and phylogenetic analysis of mitochondrial genomes
}

\author{
Yuan $\mathrm{Wu}^{\dagger 1,2}$, Jian Yang ${ }^{\dagger 2}$, Fan Yang ${ }^{2}$, Tao Liu ${ }^{2}$, Wenchuan Leng ${ }^{2}$, \\ Yonglie Chu*1 and Qi Jin*2
}

\begin{abstract}
Address: ${ }^{1}$ Department of Microbiology and Immunology, Medical School of Xi'an Jiaotong University, Shaanxi, 710061, PR China and ${ }^{2}$ State Key Laboratory for Molecular Virology and Genetic Engineering, Institute of Pathogen Biology, Chinese Academy of Medical Sciences, Beijing 100730, PR China

Email: Yuan Wu - doctorjenny@yahoo.cn; Jian Yang - yang@chgb.org.cn; Fan Yang - ymf129@163.com; Tao Liu - liutao0508@yahoo.com.cn; Wenchuan Leng - Lengwenchuan@gmail.com; Yonglie Chu* - yonglie_chu@163.com; Qi Jin* - zdsys@sina.com

* Corresponding authors †Equal contributors
\end{abstract}

Published: 21 May 2009

BMC Genomics 2009, 10:238 doi:10.1186/147|-2164-10-238
Received: I December 2008

Accepted: 2I May 2009

This article is available from: http://www.biomedcentral.com/I47I-2/64/I0/238

(c) 2009 Wu et al; licensee BioMed Central Ltd.

This is an Open Access article distributed under the terms of the Creative Commons Attribution License (http://creativecommons.org/licenses/by/2.0), which permits unrestricted use, distribution, and reproduction in any medium, provided the original work is properly cited.

\begin{abstract}
Background: Dermatophytes are fungi that cause superficial infections of the skin, hair, and nails. They are the most common agents of fungal infections worldwide. Dermatophytic fungi constitute three genera, Trichophyton, Epidermophyton, and Microsporum, and the evolutionary relationships between these genera are epidemiologically important. Mitochondria are considered to be of monophyletic origin and mitochondrial sequences offer many advantages for phylogenetic studies. However, only one complete dermatophyte mitochondrial genome (E. floccosum) has previously been determined.

Results: The complete mitochondrial DNA sequences of five dermatophyte species, $T$. rubrum (26,985 bp), T. mentagrophytes (24,297 bp), T. ajelloi ( 28,530 bp), M. canis (23,943 bp) and M. nanum $(24,105$ bp) were determined. These were compared to the $E$. floccosum sequence. Mitochondrial genomes of all 6 species were found to harbor the same set of genes arranged identical order indicating that these dermatophytes are closely related. Genome size differences were largely due to variable lengths of non-coding intergenic regions and the presence/absence of introns. Phylogenetic analyses based on complete mitochondrial genomes reveals that the divergence of the dermatophyte clade was later than of other groups of pathogenic fungi.

Conclusion: This is the first systematic comparative genomic study on dermatophytes, a highly conserved and recently-diverged lineage of ascomycota fungi. The data reported here provide a basis for further exploration of interrelationships between dermatophytes and will contribute to the study of mitochondrial evolution in higher fungi.
\end{abstract}

\section{Background}

Dermatophytes are parasitic fungi that infect skin, hair and nails of both humans and animals. They are the primary causative agents of dermatophytosis, a major public health concern in some geographic regions[1-3]. While not fatal, dermatophyte infections cause significant morbidity and are of significant cost to society because of their chronic nature and resistance to therapy. Dermatophytes encompass 3 genera, Trichophyton, Epidermophyton, and Microsporum. T. rubrum, T. mentagrophytes and M. canis are 
the most common species in hospital isolates (72$95 \%)[4,5]$. Morbidity is less commonly associated with M. nanum while T. ajelloi is a geophilic fungus that only rarely infects human.

Mitochondria are generally accepted as descendants of endosymbiotic alpha-proteobacteria[6,7] and are considered to be of monophyletic origin[8-10]. As vital physiological processes and basic adaptive strategies do not always correlate with trees derived from ribosomal sequences[11,12], mitochondrial DNA (mtDNA) sequences have become a popular tool for phylogenetic studies. Individual gene sequences often contain a limited number of informative sites and can lead to incongruent phylogenetic trees. In contrast, entire mitochondrial genomes tend to produce reliable phylogenetic trees[1113]. Despite the emergence of new technologies for rapid DNA sequence determination, sequencing of complete mtDNAs is still more feasible and economical than whole-genome sequencing. Furthermore, complete mtDNA sequences reveal gene content, order and position, and provide further information regarding introns and intergenic regions [10].

The number of mitochondrial genomes sequenced has increased greatly over the past decade, notably through the interdisciplinary collaboration of the Organelle Genome Megasequencing Program http:// megasun.bch.umontreal.ca/ogmp. Some thousands of complete mtDNA sequences are already available from taxonomically diverse organisms including fungi, plants and animals. This resource provides an unprecedented insights into the origin and evolution of the mitochondrial genome [13]. In comparison to the genomes of freeliving alpha-proteobacteria, the number of genes contained within the modern mitochondrial genome has been greatly reduced. It is inferred that many previously functional genes have been transferred to the nucleus; others appear to have been replaced by pre-existing nuclear genes of similar function [14]. Moreover, recent studies have suggested that positive selection plays a role in mitochondrial evolution [15-17] while mtDNA polymorphisms are thought to be maintained within populations via selection on the joint mitochondrial-nuclear genotype [15].

The fungal mitochondrial genome typically contains 14 conserved protein-coding genes, 22-26 tRNA genes, and 2 rRNA genes[9,18-20]. mtDNA divergence between different fungal species is predominantly associated with variation in intergenic regions, intronic sequences, and gene order[13,21]. The Fungal Mitochondrial Genome Project was launched over a decade ago [22] and more than 50 complete mitochondrial genomes of fungi have been determined to date (available from GenBank website
http://www.ncbi.nlm.nih.gov/genomes/Genomes-

Group.cgi?taxid $=4751$ \&opt $=$ organelle). Nevertheless, only one sequence is derived from a dermatophyte, i.e. E. floccosum [12]. Indeed, the dearth of publicly available genomic data is a major barrier to biomedical research on dermatophytes [23].

We report here complete mtDNA sequences for 5 dermatophytes including 3 species of Trichophyton (T. rubrum, $T$. mentagrophytes and T. ajelloi) and 2 species of Microsporum (M. canis and M. nanum). These sequences, with the previously reported $E$. floccosum $\mathrm{mtDNA}$ sequence [12], have permitted systematic comparative analysis of dermatophytes. The mitochondrial genomes of dermatophytes are highly conserved, indicating that these superficial fungi are closely related. Furthermore, phylogenetic analysis based on complete mitochondrial genomes has revealed that dermatophytes arose very late among the ascomycota fungi. This is the first comparative genomic study on dermatophytes and will provide valuable insights into the genomics and phylogeny of this important group of fungal pathogens.

\section{Results and discussion General features}

The mitochondrial sequences of $T$. rubrum, T. mentagrophytes, T. ajelloi, M. canis and M. nanum are circular DNAs of $26,985 \mathrm{bp}, 24,297 \mathrm{bp}, 28,530 \mathrm{bp}, 23,943 \mathrm{bp}$ and 24,105 bp, respectively (Fig. 1). The mitochondrial genome of $M$. canis is the smallest of known dermatophytes, while the previously determined E. floccosum is the largest and exceeds $30 \mathrm{~kb}$ [12]. The size differences between mitochondrial genome sizes are primarily due to interspecific variation in intergenic regions and introns (see below).

With the exception of T. rubrum and E. floccosum that carry several additional hypothetical genes, each genome encodes 15 conserved protein-coding genes, 2 rRNA genes ( $r n l$ and $r n s$ ), and 25 tRNA genes (Table 1, Fig. 1). The dermatophyte mitochondrial genomes are highly compact: structural genes account for $>70 \%$ of each genome (Table 1 ). All genes are encoded on the same DNA strand. Moreover, all the mitochondrial genomes retain colinearity at the nucleotide level without detectable DNA rearrangement (Fig. 2). The high conservation of genome structure indicates that these dermatophytes are closely related.

The overall G+C content of the 5 genomes is $~ 24 \%$ (Table 1) consistent with the characteristic AT-rich nature of fungal mitochondrial genomes. The $\mathrm{G}+\mathrm{C}$ content of genomic regions encoding RNA genes is usually higher than the genome average (Table 1) while the majority of proteincoding genes, with exception of cox1, have a lower $\mathrm{G}+\mathrm{C}$ content (Fig. 1). The unusual $\mathrm{G}+\mathrm{C}$ content of cox1 may 




Figure I

Circular representations of the dermatophyte mitochondrial genomes. Protein-coding genes are represented by purple arrows. rRNA and tRNA genes are indicated by green blocks and blue triangles respectively. $\mathrm{G}+\mathrm{C}$ contents with a window size of $\mathrm{I} \mathrm{kb}$ are shown in yellow (higher than genome average) or orange (lower than average) curves. The inner scale is marked at $5 \mathrm{~kb}$ intervals.

reflect its unusual location sandwiched between 2 tRNA gene clusters.

\section{Protein-coding genes and rRNAs}

The five mitochondrial genomes share 15 protein-coding genes. These include ATP-synthase subunits 6, 8, and 9 (atp6, atp8, and atp9), cytochrome oxidase subunits I, II, and III (cox $1, \operatorname{cox} 2$, and cox3), apocytochrome b (cob), a ribosomal protein (rps5), and NADH dehydrogenase subunits (nad1, nad2, nad3, nad4, nad4L, nad5, and nad6). T. rubrum carries 2 additional hypothetical genes (orf1 and orf2) located between tRNA-Val and tRNA-Met (Fig. 1). Both genes have homologs of $>90 \%$ nucleotide similarity in the previously determined E. floccosum mitochondrial genome (GenBank: AY916130). The function of these putative genes is not yet known; further studies will be required to determine whether they play a role in mitochondrial function.
Synonymous base substitutions (dS) are considered to be selectively neutral while substitutions causing amino acid substitution, or non-synonymous substitutions ( $\mathrm{dN})$, are almost always adaptive mutations. The $\mathrm{dN} / \mathrm{dS}$ ratio therefore affords an index of selective pressures operating on protein-coding genes. Because selection for adaptive amino acid substitutions increases $\mathrm{dN}$, a dN/dS ratio of $>1$ is found in genes subject to selection for change (positive selection). In contrast, a $\mathrm{dN} / \mathrm{dS}$ ratio of $<1$ indicates conservation of essential amino acid sequences (negative selection). We calculated the $\mathrm{dN} / \mathrm{dS}$ ratio for each of the 15 dermatophyte protein-coding gene. This revealed that all genes are subject to strong negative selection; no statistically significant sites of positive selection were found (see Additional file 1).

The genomic organization of the 15 conserved proteincoding genes in mtDNA is surprisingly identical across all 




Figure 2

Pairwise linear genome comparisons of dermatophyte $m$ tDNAs. Protein-coding genes are represented by purple arrows. rRNA and tRNA genes are shown by blue blocks and triangles respectively. Red parallelograms indicate the locations of homologous genomic regions in adjacent genome pairs.

Table I: General features of the mitochondrial genomes of dermatophytes

\begin{tabular}{|c|c|c|c|c|c|c|}
\hline Species & T. rubrum & T. mentagrophytes & T. ajelloi & M. canis & M. nanum & E. floccosum* \\
\hline Genome size (bp) & 26,985 & 24,297 & 28,530 & 23,943 & 24,105 & 30,910 \\
\hline $\mathrm{G}+\mathrm{C}$ content $(\%)$ & 23.51 & 24.03 & 23.45 & 24.15 & 24.47 & 23.43 \\
\hline No. of protein-coding genes & 17 & 15 & 15 & 15 & 15 & 24 \\
\hline G G $+C$ content of protein-coding genes (\%) & 22.17 & 22.30 & 22.38 & 22.79 & 22.94 & 22.00 \\
\hline No. of rRNAs/tRNAs & $2 / 25$ & $2 / 25$ & $2 / 25$ & $2 / 25$ & $2 / 25$ & $2 / 25$ \\
\hline $\mathrm{G}+\mathrm{C}$ content of RNA genes (\%) & 32.64 & 32.65 & 32.85 & 32.61 & 32.55 & 32.88 \\
\hline Percentage of structural genes (\%) & 79.72 & 83.01 & 70.34 & 84.09 & 83.76 & 87.37 \\
\hline No. of introns & 1 & 1 & 2 & $\mathrm{I}$ & I & 5 \\
\hline No. of intronic ORFs & I & I & I & I & I & 5 \\
\hline
\end{tabular}

*Data from GenBank record AY916130. 
the known dermatophytes (Fig. 2). Furthermore, the same gene order is also largely conserved in other pathogenic filamentous fungi such as Penicillium marneffei and Aspergillus niger that cause invasive infections [12]. However, the order of these conserved genes is markedly rearranged in the mtDNA of the opportunistic pathogen Candida albicans [24] (Fig. 3). The similarity of mtDNA gene order between different fungal species was found to correlate with their evolutionary relationships inferred from phylogenetic analysis (see below).

The large ( $r n l)$ and small (rns) ribosomal RNA genes are present in all dermatophyte mitochondrial genomes at the same relative locations. The rps5 gene encodes a ribosomal protein that may play a role in maintaining the integrity of the mitochondrial genome [25]; this gene is present within the intron of $r n l$ as expected. The intronic location of this ribosomal protein is therefore maintained in all known dermatophyte mitochondrial genomes.

The previously described nad4L-nad5 consecutive gene unit was observed in all 5 mitochondrial genomes. This gene pair is believed to be present in all ascomycota as well as the basidiomycota and zygomycota, but is interrupted by one or more genes in chytridiomycota. This may reflect early phylogenetic divergence of these fungi from the ascomycota [26]. Interestingly, the additional continuous gene unit, nad4L-nad5-nad2, characterized by one base overlap and no interruption in gene junctions, is shared by all dermatophyte mitochondrial genomes with the exception of the 2 earlier divergent species, M. canis and T. ajelloi (see phylogenetic section below). The arrangement of such uninterrupted blocks may reflect strong conservation during evolution from a common ancestor [27]; more dermatophytes may be expected to carry the consecutive nad4L-nad5-nad2 gene unit.

\section{tRNAs and codon usage}

All 5 mitochondrial genomes encode the same set of 25 tRNA species with the potential to deliver all 20 amino acids. Multiple tRNA isoacceptors exist for only leucine, serine, arginine and methionine. Interestingly, the organization of the 25 tRNA genes is conserved between all the mitochondrial genomes. The majority of tRNAs ( 23 of 25) are grouped into 3 clusters flanked by the atp8-rnl-cox1atp9 gene cluster and containing 8, 3 and 12 tRNAs, respectively (Fig. 1). The exceptions are T. rubrum and $E$. floccosum where the largest tRNA cluster between $\mathrm{rnl}$ and cox1 is interrupted and subdivided into two sub-clusters by a $2.5 \mathrm{~kb}$ DNA fragment containing additional hypothetical genes (Fig. 2).

tRNA genes have been proposed to play a role in gene shuffling as they are located between protein-coding genes and can act as mobile elements [28]. Homology between isolated tRNA genes may permit genetic recom- bination, and therefore genetic rearrangement, while gene shuffling is less likely to take place via recombination between conserved clusters of tRNA genes[29,30]. The low number of isolated tRNA genes ( 2 of 25) may therefore contribute to the high conservation of dermatophyte mtDNA.

All mitochondrial protein-coding genes commence with a classical methionine codon (ATG) and terminate with TAA, the preferred fungal mitochondrial termination codon [31]. The only exception is atp9 where TGA is the translation stop codon. Table 2 summarizes codon usages for all protein-coding genes in the 5 mitochondrial genomes. The most frequently used codons are TTA (Leu), ATA (Ile), and TTT (Phe), accounting for about one third of all codons, and indicating a clear preference for amino acids with nonpolar side chains. This is likely to reflect the fact that the majority of the encoded polypeptides are integral membrane proteins. As expected from the AT-rich nature of dermatophyte mtDNA, the third position of the most frequently used codons is strongly biased towards A or $\mathrm{T}$ (Table 2). Furthermore, the most rarely-used codons, appearing no more than 5 times in all genomes (TGC, TGG, GGC, CTG, CTC, AGG, CGA, CCG, CCC and ACC), or absent (CGC, CGG, and ACG), all contain 2 or more G/ $\mathrm{C}$ nucleotides in each codon.

\section{Intergenic regions and introns}

The dermatophyte genomes are highly compact with short intergenic regions. In M. canis there is no intergenic region $>1 \mathrm{~kb}$, while only one intergenic region exceeds 1 $\mathrm{kb}$ in the other genomes. Because protein-coding genes and RNAs are highly conserved between species, the differences in mitochondrial genome sizes between the different dermatophytes are largely explained by length variation in intergenic regions. The longest intergenic region in M. canis is only $919 \mathrm{nt}$ while all other intergenic regions in this species are under $500 \mathrm{nt}$. In contrast, T. ajelloi mtDNA contains 4 long intergenic regions (>500 bp) and the longest (located at the nad2-cob gene junction) spans $>2.5 \mathrm{~kb}$ (Fig. 1). This divergence in intergenic regions explains the $>4 \mathrm{~kb}$ difference in the genome sizes of T. ajelloi and M. canis.

mtDNA intergenic regions diverge not only in size but also in sequence. Indeed, multiple pairwise alignment of the different genomes indicates that colinearities are interrupted almost exclusively in intergenic regions (Fig. 2). However, many intergenic regions were found to be conserved between T. rubrum and T. mentagrophytes, consistent with the close evolutionary relationship of these 2 species as revealed by phylogenetic analysis (Fig. 4). Remarkably, the longest intergenic region in all genomes is located between $c o b$ and nad3 (with the exception of $T$. ajelloi; see above), but pairwise alignment revealed little similarity between the cob-nad3 intergenic sequences of 


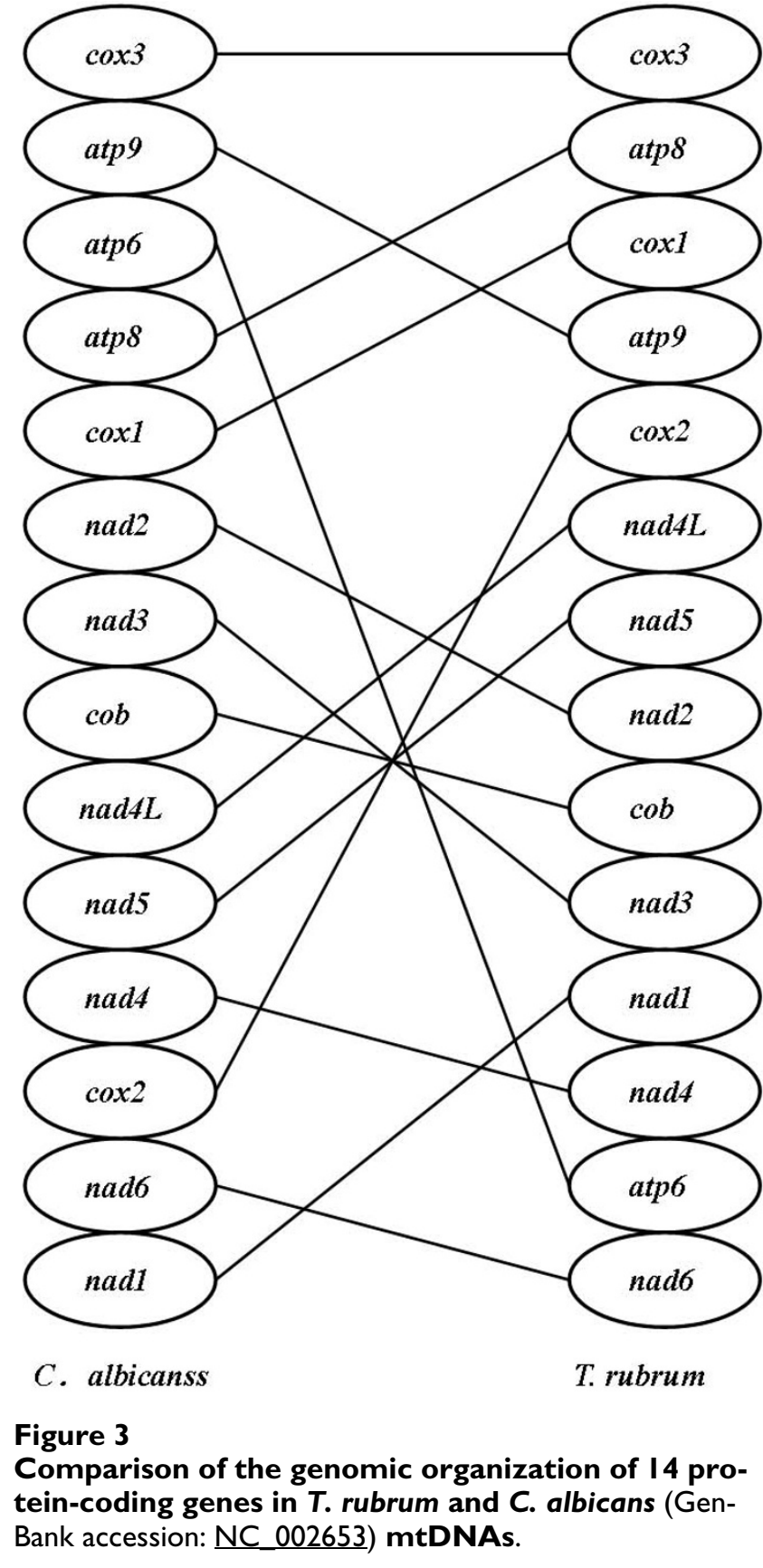

the different species as indicated by the blank space in the linear comparison view (Fig. 2). Such divergent intergenic regions may serve as potential genetic markers for species/ strain identification of dermatophytes.

Many fungal mitochondrial genomes are intron-rich [32]. In contrast, all the dermatophyte mtDNA gene sequences harbor a single intron (within the $r n l$ gene), with the exception of T. ajelloi that carries an additional group I intron (801 bp) within nad1 (Fig. 1). However, previous mtDNA sequence studies revealed that another strain of $T$. rubrum (IP1817.89) harbors 2 additional introns within nad1 and atp9, respectively [33]. Moreover, the E. floccosum mitochondrial genome carries 4 additional introns located within in $\operatorname{cox} 1$, nad5 (2 introns) and $c o b$ [12]. The number of introns is therefore strain-specific and also contributes to variation in mitochondrial genome size.

\section{Phylogenetic analysis}

Broad phylogenetic trees of fungi were previously constructed based on rDNA [34-36] or nuclear protein-coding genes $[37,38]$ but these studies did not permit the elucidation of higher-order relationships. A combination of 6 gene regions was recently employed to construct a fungal phylogenetic tree comprising 200 species [39]. Unfortunately, no dermatophytes were included in this study. We therefore performed phylogenetic analysis based on the complete mitochondrial genomes of 35 species of ascomycota, including 6 dermatophytes, 12 other filamentous fungi, and 17 yeasts (Fig. 4). The high bootstrap values of most nodes indicate the robustness of the tree computed. Fungal species of ascomycota are clustered into 3 distinct groups corresponding to subphyla Pezizomycotina (filamentous fungi), Saccharomycotina (budding yeast) and Taphrinomycotina (fission yeast) respectively (Fig. 4). This confirmed the reliability of mtDNA sequences in fungal phylogenetic analysis.

Interestingly, the tree reported here divides the clade of filamentous fungi into 2 subgroups (Fig. 4). With only a few exceptions, the dermatophytes cluster together with invasive pathogenic fungi of humans and animals, while the other filamentous fungi, mostly pathogens of plants or insects, form a parallel branch (Fig. 4). This suggests that host adaptation has driven the evolution of filamentous fungi. Indeed, previous phylogenetic studies revealed separation between anthropophilic and geophilic species of Trichophyton [40] suggesting that ecology is a particularly strong driver of dermatophyte evolution [41].

In the tree established here all the dermatophytes species clustered into a single branch, confirming the monophyletic origin of the dermatophyte lineage. Aspergillus [42] and P. marneffei [43] comprise a separate branch that shares an immediate ancestor with the dermatophyte group (Fig. 4). However, the 2 sister branches of human pathogenic fungi (causing superficial and invasive infections respectively) are represented by distinct patterns in the phylogenetic tree. The dermatophytes as a group show far less divergence but longer ancestral branch than the Aspergillus-Penicillium clade (Fig. 4). This indicates the divergence from the latest common ancestor of dermatophytes was later than the Aspergillus-Penicillium group.

Fossil evidence has allowed dating of the emergence of the ascomycota [44]. Based on this calibration, the dermatophyte lineage may be estimated to have diverged from other fungi at about 32 million years ago (Ma). This result 
Table 2: Codon usage in protein-coding genes of $T$. rubrum, T. mentagrophytes, $T$. ajelloi, $M$. canis and $M$. nanum

\begin{tabular}{|c|c|c|c|c|c|c|}
\hline Codon & AA & M. canis & M. nanum & T. ajelloi & T. mentagrophytes & T. rubrum \\
\hline $\mathrm{AAA}$ & Lys (K) & 173 & 158 & 168 & 170 & 209 \\
\hline \multirow[t]{2}{*}{ AAG } & Lys (K) & 2 & 9 & 9 & 3 & 8 \\
\hline & Total K & 175 & 167 & 177 & 173 & 217 \\
\hline AAC & Asn $(\mathrm{N})$ & 12 & 20 & 15 & 10 & 17 \\
\hline \multirow[t]{2}{*}{ AAU } & Asn $(\mathrm{N})$ & 256 & 259 & 246 & 262 & 307 \\
\hline & Total $\mathrm{N}$ & 268 & 279 & 261 & 272 & 324 \\
\hline$A C A$ & $\operatorname{Thr}(\mathrm{T})$ & 139 & 129 & 126 & 136 & 146 \\
\hline $\mathrm{ACC}$ & $\operatorname{Thr}(\mathrm{T})$ & 0 & 0 & I & 0 & 0 \\
\hline ACG & $\operatorname{Thr}(\mathrm{T})$ & 0 & 0 & 0 & 0 & 0 \\
\hline \multirow[t]{2}{*}{$\mathrm{ACU}$} & $\operatorname{Thr}(\mathrm{T})$ & 92 & 100 & 102 & 97 & 101 \\
\hline & Total T & 231 & 229 & 229 & 233 & 247 \\
\hline AGC & Ser (S) & 7 & 6 & 2 & 5 & 6 \\
\hline \multirow[t]{2}{*}{ AGU } & $\operatorname{Ser}(S)$ & 144 & 149 & 154 & 152 & 165 \\
\hline & Total S & 151 & 155 & 156 & 157 & $17 \mid$ \\
\hline$A \cup A$ & Ile (I) & 357 & 353 & 336 & 379 & 394 \\
\hline AUC & Ile (I) & 12 & 18 & 11 & 8 & 19 \\
\hline \multirow[t]{2}{*}{ AUU } & Ile (I) & 207 & 209 & 247 & 203 & 231 \\
\hline & Total I & 576 & 580 & 594 & 590 & 644 \\
\hline \multirow[t]{2}{*}{ AUG } & Met (M) & 114 & 117 & 108 & 116 & 124 \\
\hline & Total M & 114 & 117 & 108 & 116 & 124 \\
\hline CAA & $\mathrm{Gln}(\mathrm{Q})$ & 95 & 94 & 91 & 100 & 105 \\
\hline \multirow[t]{2}{*}{ CAG } & $\mathrm{Gln}(\mathrm{Q})$ & 4 & 4 & 4 & I & 3 \\
\hline & Total Q & 99 & 98 & 95 & 101 & 108 \\
\hline CAC & His $(H)$ & 12 & 9 & 5 & 8 & 8 \\
\hline \multirow[t]{2}{*}{ CAU } & His $(H)$ & 70 & 70 & 77 & 70 & 78 \\
\hline & Total H & 82 & 79 & 82 & 78 & 86 \\
\hline $\mathrm{CCA}$ & Pro (P) & 46 & 53 & 38 & 55 & 60 \\
\hline $\mathrm{CCC}$ & Pro (P) & 2 & 0 & I & 0 & I \\
\hline
\end{tabular}


Table 2: Codon usage in protein-coding genes of T. rubrum, T. mentagrophytes, T. ajelloi, $M$. canis and $M$. nanum (Continued)

\begin{tabular}{|c|c|c|c|c|c|c|}
\hline CCG & Pro (P) & 2 & 3 & 0 & 0 & 0 \\
\hline \multirow[t]{2}{*}{$\mathrm{CCU}$} & Pro (P) & 98 & 91 & 106 & 91 & 94 \\
\hline & Total P & 148 & 147 & 145 & 146 & 155 \\
\hline CGA & $\operatorname{Arg}(\mathrm{R})$ & 0 & I & 3 & 0 & 1 \\
\hline CGC & $\operatorname{Arg}(\mathrm{R})$ & 0 & 0 & 0 & 0 & 0 \\
\hline CGG & $\operatorname{Arg}(\mathrm{R})$ & 0 & 0 & 0 & 0 & 0 \\
\hline AGA & $\operatorname{Arg}(\mathrm{R})$ & 82 & 83 & 86 & 82 & 91 \\
\hline AGG & $\operatorname{Arg}(\mathrm{R})$ & 0 & 0 & 0 & 0 & 1 \\
\hline \multirow[t]{2}{*}{$\mathrm{CGU}$} & $\operatorname{Arg}(\mathrm{R})$ & 12 & 9 & 9 & 9 & 10 \\
\hline & Total R & 94 & 93 & 98 & 91 & 103 \\
\hline CUA & Leu (L) & 19 & 13 & 16 & 13 & 14 \\
\hline CUC & Leu (L) & I & 0 & 0 & 0 & 0 \\
\hline CUG & Leu (L) & I & 0 & I & I & 1 \\
\hline UUA & Leu (L) & 655 & 666 & 658 & 673 & 705 \\
\hline UUG & Leu (L) & 10 & 13 & 12 & 7 & 9 \\
\hline \multirow[t]{2}{*}{ CUU } & Leu (L) & 43 & 41 & 53 & 34 & 42 \\
\hline & Total L & 729 & 733 & 740 & 728 & 771 \\
\hline GAA & Glu (E) & 97 & 104 & 105 & 104 & 125 \\
\hline \multirow[t]{2}{*}{ GAG } & Glu (E) & 7 & 7 & 4 & 6 & 8 \\
\hline & Total E & 104 & 111 & 109 & 110 & 133 \\
\hline GAC & Asp (D) & 5 & 2 & 5 & 4 & 5 \\
\hline \multirow[t]{2}{*}{ GAU } & Asp (D) & 112 & 111 & 108 & 113 & $14 \mid$ \\
\hline & Total D & 117 & 113 & 113 & 117 & 146 \\
\hline GCA & Ala (A) & 62 & 63 & 63 & 64 & 64 \\
\hline GCC & Ala (A) & 6 & 2 & 2 & 2 & 3 \\
\hline GCG & Ala (A) & 2 & 5 & 0 & 2 & 1 \\
\hline \multirow[t]{2}{*}{ GCU } & Ala (A) & 152 & 155 & 156 & 153 & 161 \\
\hline & Total A & 222 & 225 & 221 & 221 & 229 \\
\hline GGA & Gly (G) & 44 & 39 & 36 & 45 & 54 \\
\hline
\end{tabular}


Table 2: Codon usage in protein-coding genes of T. rubrum, T. mentagrophytes, T. ajelloi, M. canis and M. nanum (Continued)

\begin{tabular}{|c|c|c|c|c|c|c|}
\hline GGC & Gly (G) & 0 & 0 & $T$ & $T$ & 1 \\
\hline GGG & Gly (G) & I & 2 & I & I & 2 \\
\hline \multirow[t]{2}{*}{ GGU } & Gly (G) & 251 & 252 & 253 & 250 & 256 \\
\hline & Total G & 296 & 293 & 291 & 297 & 313 \\
\hline GUA & Val (V) & 125 & 124 & 113 & 125 & 140 \\
\hline GUC & Val (V) & I & 1 & 2 & 1 & I \\
\hline GUG & Val (V) & 8 & 12 & 6 & 3 & 2 \\
\hline \multirow[t]{2}{*}{ GUU } & $\mathrm{Val}(\mathrm{V})$ & 130 & 122 & 133 & 122 & 124 \\
\hline & Total V & 264 & 259 & 254 & 251 & 267 \\
\hline UAA & $\operatorname{Ter}()$. & 13 & 13 & 12 & 13 & 14 \\
\hline \multirow[t]{2}{*}{ UAG } & Ter (.) & I & I & 2 & I & 2 \\
\hline & Total. & 14 & 14 & 14 & 14 & 16 \\
\hline UAC & Tyr (Y) & 13 & 25 & 14 & 18 & 22 \\
\hline \multirow[t]{2}{*}{ UAU } & Tyr (Y) & 227 & 217 & 229 & 224 & 248 \\
\hline & Total Y & 240 & 242 & 243 & 242 & 270 \\
\hline UCA & $\operatorname{Ser}(S)$ & 148 & 142 & 133 & 148 & 149 \\
\hline UCC & Ser $(S)$ & 2 & 2 & 2 & 1 & 2 \\
\hline UCG & Ser $(S)$ & 0 & I & I & I & 3 \\
\hline \multirow[t]{2}{*}{ UCU } & Ser $(S)$ & 127 & 136 & 140 & 133 & 138 \\
\hline & Total S & 277 & 281 & 276 & 283 & 292 \\
\hline UGA & $\operatorname{Trp}(\mathrm{W})$ & 61 & 62 & 60 & 61 & 63 \\
\hline \multirow[t]{2}{*}{ UGG } & $\operatorname{Trp}(\mathrm{W})$ & 1 & 0 & 2 & I & 1 \\
\hline & Total W & 62 & 62 & 62 & 62 & 64 \\
\hline UGC & Cys (C) & 1 & 0 & 0 & 0 & 0 \\
\hline \multirow[t]{2}{*}{ UGU } & Cys (C) & 31 & 32 & 35 & 30 & 37 \\
\hline & Total C & 32 & 32 & 35 & 30 & 37 \\
\hline UUU & Phe (F) & 339 & 323 & 358 & 346 & 377 \\
\hline \multirow[t]{2}{*}{ UUC } & Phe (F) & 65 & 71 & 42 & 54 & 65 \\
\hline & Total F & 404 & 394 & 400 & 400 & 442 \\
\hline
\end{tabular}




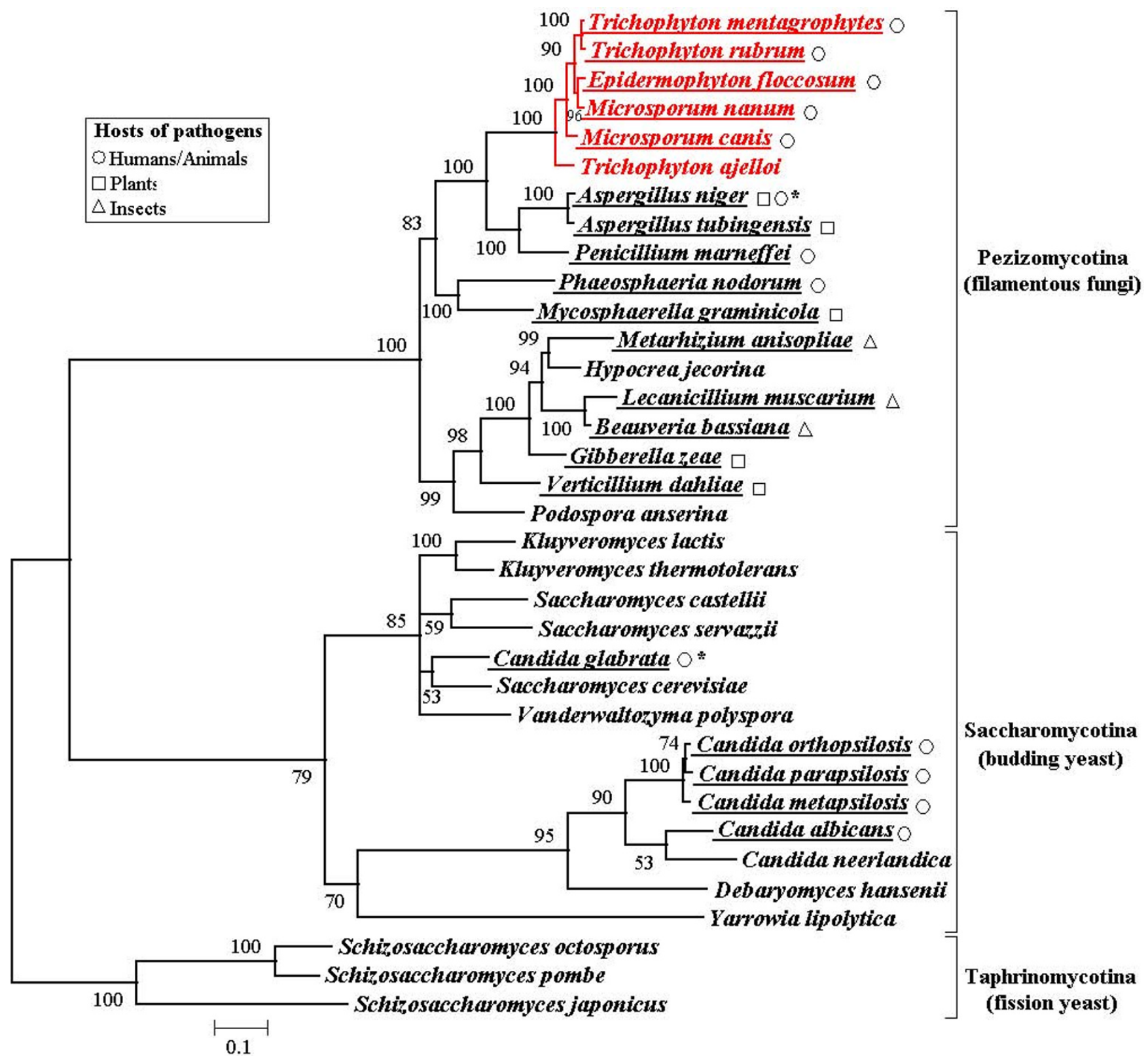

Figure 4

Maximum likelihood phylogenetic tree based on concatenated mitochondrial proteins. A total of 4,298 amino acid positions were used for the inference using the TREE-PUZZLE program. Additional sequences obtained from GenBank were: Epidermophyton floccosum (AY916130), Aspergillus niger (NC 007445), Aspergillus tubingensis (NC 007597), Penicillium marneffei (NC_005256), Phaeosphaeria nodorum (NC 009746), Mycosphaerella graminicola (NC 010222), Metarhizium anisopliae (NC 008068), Hypocrea jecorina (NC 003388), Lecanicillium muscarium (NC 0045I4), Beauveria bassiana (NC 010652), Gibberella zeae (NC 009493), Verticillium dahliae (NC 003060), Podospora anserina (NC 00I329), Kluyveromyces lactis (NC 006077), Kluyveromyces thermotolerans ( $\mathrm{NC}$ 006626), Saccharomyces castellii (NC 003920), Saccharomyces servazzii (NC 004918), Candida glabrata (NC 00469I), Saccharomyces cerevisiae (NC 001224), Vanderwaltozyma polyspora (NC 009638), Candida orthopsilosis (NC 006972), Candida parapsilosis (NC 005253), Candida metapsilosis ( NC 006971), Candida albicans (NC 002653), Candida neerlandica (NC_0III33), Debaryomyces hansenii (NC_010166), Yarrowia lipolytica (NC_002659), Schizosaccharomyces octosporus (NC 0043 12), Schizosaccharomyces pombe (NC 001326), Schizosaccharomyces japonicus (NC 004332). Bootstrap values obtained in 100 replicates are indicated at the nodes. Dermatophytes are highlighted in red, and pathogenic fungal species are underlined; symbols following indicate their respective hosts (see legend). *A. niger are mainly pathogenic to plants but can infect people if large amounts of spores are inhaled. C. glabrata only infect person with lower immunity. 
is consistent with a previous rough estimate ( $50 \mathrm{Ma}$ ) based on nucleotide substitution rates in the small ribosomal subunit RNA [45]. However, the timing of the radiation of the dermatophytes is much later than the divergence of Candida and Saccharomyces at $723 \mathrm{Ma}$ as previously estimated using 20-188 protein sequences [46]. The high conservation of the dermatophyte mitochondrial genome also suggests that the different dermatophytes diverged only recently.

Conventional phenotypic taxonomy has divided the dermatophytes into 3 genera: Trichophyton, Microsporum and Epidermophyton [47]. Though only a limited number of dermatophyte species were included in the present study, the phylogenetic tree established here does not follow this genus demarcation (Fig. 4). Indeed, recent molecular phylogenetic studies have revealed that both Trichophyton and Microsporum are paraphyletic [48], prompting reevaluation of the phylogenetic relationships between different dermatophytes [41]. Remarkably, the divergence of T. ajelloi from the inferred common ancestor was much earlier than of the other dermatophyte species (Fig. 4). This is consistent with the geophilic features of T. ajelloi: the soil environment may have afforded an early ecological niche for all dermatophyte species prior to more recent adaptation to specialized hosts including animals and humans. An earlier study based on 25S rRNA sequences reported that T. ajelloi and T. terrestre (not included in the present study) are separated from the 'true dermatophyte' [49] and further support the suggestion that Microsporum, as well as the zoophilic and anthropophilic Trichophyton species, evolved from a geophilic member of Trichophyton [48].

\section{Conclusion}

Previous studies into the evolutionary relationships between dermatophyte species have been based on nuclear ribosomal internal transcribed spacers (ITS)[40,50,51], large ribosomal RNA subunits (LSU) [49], chitin synthase (CHS) [52-54] and DNA topoisomerase II genes [55], as well as on PCR fingerprinting [56] and restriction fragment length polymorphism (RFLP) [57] analysis of mitochondrial DNA. The dermatophytes were found to constitute a homogeneous group of species with low genetic diversity contrasting with high phenotypic heterogeneity $[41,58]$. We now report comparative analysis of 6 complete mitochondrial genomes from all 3 dermatophyte genera (Trichophyton, Microsporum and Epidermophyton). The composition and organization of genes within the mtDNAs of all dermatophytes analyzed was found to be substantially identical, reinforcing the view that dermatophytes are closely related and constitute a highly conserved lineage of filamentous fungi.

Comparative genomics provides a powerful tool for uncovering similarities and differences between species.
The present study represents the first application of systematic comparative genomics to dermatophyte phylogeny. The common features shared by all (or the majority) of dermatophyte mitochondrial genomes are as follows. 1. Retention of genome colinearity with high nucleotide sequence similarity ( $>90 \%$ ) in coding regions; differences between species are largely restricted to introns and intergenic regions. 2. Strict conservation of the nad4L-nad5 gene unit; the consecutive nad4L-nad5-nad2 gene unit is also probably present in most dermatophytes. 3 . The presence of 3 tRNA gene clusters of identical composition with 2 isolated tRNA genes at identical locations in all dermatophyte mtDNAs. 4. A characteristic rarity of intronic sequences compared to other fungal species.

Phylogenetic analysis has confirmed the monophyletic origin of dermatophytic fungi; these form a distinct clade among filamentous fungi. Compared with other pathogenic fungi such as those causing invasive infections, dermatophytes comprise a closely-related and recentlydiverged lineage of ascomycota fungi. The genomic data presented here will allow further exploration of the relationships between different dermatophyte species and will be of general utility in the study of mitochondrial evolution in higher fungi.

\section{Methods}

\section{Strains and DNA preparation}

Type strains of T. ajelloi (ATCC 28454),M. canis (ATCC 36299), and M. nanum (ATCC 42129) and clinical strains of T. rubrum (BMU01672) and T. mentagrophytes (BMU03104) were kindly provided by Ruoyu Li (Research Center for Medical Mycology, Peking University, Beijing, China). All strains were confirmed by ribosomal ITS sequencing and comparison with the NCBI nucleotide database.

Culture conditions and harvesting of mycelia were as described previously [59]. Mycelia were ground to powder in liquid nitrogen and samples were transferred to liquid nitrogen-cooled $15 \mathrm{ml}$ falcon tubes. Two volumes of lysis buffer 1 (0.35 M sucrose, $50 \mathrm{mM} \mathrm{Na}{ }_{2}$ EDTA, $20 \mu \mathrm{g} / \mathrm{ml}$ proteinase $\mathrm{K}, 10 \mathrm{mM}$ Tris.HCl pH 7.4) were added and the mixture were vortexed vigorously for $10 \mathrm{~min}$ at $4{ }^{\circ} \mathrm{C}$. The lysate was held on ice for $15 \mathrm{~min}$ and debris was removed by centrifugation at $3,500 \mathrm{rpm}$ for $10 \mathrm{~min}$ at $4{ }^{\circ} \mathrm{C}$ ). Organelles were collected by centrifugation $(30,000 \mathrm{rpm}$, $\left.1 \mathrm{~h}, 4^{\circ} \mathrm{C}\right)$ and resuspended into buffer $2(150 \mathrm{mM} \mathrm{NaCl}$, $50 \mathrm{mM} \mathrm{Na}{ }_{2}$ EDTA, $20 \mu \mathrm{g} / \mathrm{ml}$ proteinase $\mathrm{K}, 10 \mathrm{mM}$ Tris. $\mathrm{HCl}$ $\mathrm{pH}$ 7.4). Total DNA was isolated using DNeasy Plant Mini Kit (QAIGEN) according to the manufacturer's instructions.

\section{DNA sequencing, assembly and annotation}

Three mtDNA sequences of T. rubrum strain IP1817.89 deposited in GenBank (Accession numbers: X65223, 
$\underline{\mathrm{X} 88896}$ and $\underline{\mathrm{Y} 98476}$ ) already covered $>80 \%$ of the mitochondrial genome[60-62]. Four pairs of primers for longdistance and accurate polymerase chain reaction (LAPCR) were designed according to the known T. rubrum sequences and their locations to the complete mitochondrial genome of $E$. floccosum to create a mtDNA sequence scaffold for $T$. rubrum strain BMU016721 (data not shown). The LA-PCR system contained $0.5 \mu \mathrm{l}$ LATaq polymerase, $5 \mu \mathrm{l} 10 \times$ LA PCR buffer, $8 \mu \mathrm{l}$ dNTP mixture, $1 \mu \mathrm{l}$ template DNA, $1 \mu \mathrm{l}$ primer $1,1 \mu \mathrm{l}$ primer 2 , and 33.5

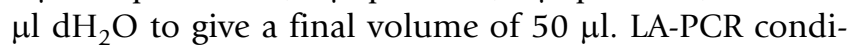
tions were $94^{\circ} \mathrm{C}, 1 \mathrm{~min} ; 98^{\circ} \mathrm{C}, 10 \mathrm{sec} ; 46.8^{\circ} \mathrm{C}, 15 \mathrm{~min}$; $72^{\circ} \mathrm{C}, 10 \mathrm{~min}$. Steps 2 and 3 were repeated for 30 cycles. All regents were from Takara. LA-PCR products were cloned into the PCR-XL-TOPO vector (Invitrogen). Recombinant plasmids were analyzed by restriction analysis to confirm the presence of the insert DNA. Primerwalking methods were then used to obtain the complete mtDNA sequence of $T$. rubrum.

The complete T. rubrum mitochondrial sequence revealed that the genomes were colinear and the overall nucleotide sequence similarity between mtDNAs of T. rubrum and $E$. floccosum was $>94 \%$, indicating that the mitochondrial genomes of these 2 species are highly conserved. Based on this observation an optimized PCR strategy was devised for mtDNA sequence determination for the other dermatophytes. A selected set of 41 primer pairs used for $T$. rubrum mtDNA sequencing was applied to the sequencing of mtDNAs from the other dermatophyte species. In about half of cases the expected specific amplicons were generated (see Additional file 2). Products of length $<2 \mathrm{~kb}$ were sequenced after purification with QIAquick Gel Extraction Kit (QAIGEN); larger amplicons were cloned into the PCR-XL-TOPO vector (Invitrogen) prior to sequencing. Further species-specific primers were designed as required for each genome in order to cover remaining sequence gaps; the complete genomes were completed by primer walking.

To confirm the authenticity of the sequences obtained, all 5 genomic sequences were confirmed by overlap PCR covering the complete mtDNA genome with direct sequencing in both directions. All sequencing was performed using an ABI3730 automated sequencer (Applied Biosystems). Sequences were assembled using the Phred/Phrap/ Consed package http://www.phrap.org/[63,64] with Phred scores set at $>20$ corresponding to an error rate $<1 \%$. The overall sequence quality of each genome was further improved by applying the following 2 criteria to each nucleotide sequenced: coverage by at least 2 independent high-quality (Phred scores $>20$ ) reads and a final consensus quality score (Phrap) of $>40$.

Potential open reading frames (ORFs) were identified using the ORF Finder program http:// www.ncbi.nlm.nih.gov/gorf/orfig.cgi based on genetic code 4. Functional annotation employed BLASTP [64] comparison of translations with the GenBank non-redundant protein database and manual curation. Ribosomal RNA genes were identified by comparison with the published rRNA sequences of E. floccosum (GenBank accession: AY916130). Transfer RNA genes were identified using the tRNAscan-SE program [65].

\section{Comparative genomics and phylogenetic analysis}

Genomic comparisons of dermatophyte mtDNAs employed GenomeComp [66]. Orthologs between the mitochondrial genomes of T. rubrum and C. albicans were identified by bidirectional BLASTP comparisons. Fourteen of the 15 conserved proteins (excluding Rps5) were used for whole mitochondrial genome-based phylogenetic analysis of 18 filamentous fungi (including 6 dermatophytes) and 17 yeasts. The sequences of the selected proteins were extracted from the fungal mitochondrial genomes in the GenBank database. Protein sequence alignment was carried out for each individual protein using ClustalW [67]. Multi-alignments were then manually checked and trimmed with BioEdit (version 6.0, by Tom Hall, Department of Microbiology, North Carolina State University, Raleigh). The Datamonkey server was used to calculate the mean $\mathrm{dN} / \mathrm{dS}$ values of protein-coding genes for dermatophytes [68].

The dataset, a concatenation of 14 proteins comprising 4,298 amino acids, was analyzed by TREE-PUZZLE software [69] to construct the maximum likelihood (ML) tree. Before tree construction, the ProTest software [70] was used to test and determine optimal model-fitting of the sequence data. The WAG model was adopted as optimal selection. The heterogeneity rate was estimated by gamma distribution with 8 rate categories and the $\alpha$-parameter was estimated from the dataset. Reliability of the dataset was assessed by bootstrap. One hundred permutation datasets were generated using the SEQBOOT program from the PHYLIP package (version 3.68, by Joe Felsenstein, Department of Genome Sciences, University of Washington, Seattle). For each of the 100 datasets a ML tree was constructed using the same parameters as described above. TREE-PUZZLE was then used with the 'consensus of user-defined trees' option to generate a consensus tree. Using the $400 \mathrm{Ma}$ ascomycota fossil [44] as a primary calibration point the dating of dermatophyte divergence was estimated using MEGA 4.0 software [71].

\section{Data accessibility}

The complete mitochondrial genome sequences reported in this paper have been deposited in the GenBank database under accessions: T. rubrum (FJ385026), T. mentagrophytes (FJ385027), T. ajelloi (FJ385028), M. nanum (FJ385029) and M. canis (FJ385030). 


\section{Authors' contributions}

YW performed the culture and isolation of mtDNA from the 5 dermatophytes, primer design and LA-PCR, plasmid construction and sequencing, data analysis, and drafting of the manuscript. JY carried out sequence assembly, genome annotation, comparative and phylogenetic analyses, and revised the manuscript. FY, TL and WL participated in primer design and LA-PCR, plasmid construction and sequencing. YC participated in the design of the Experiment Proposal and revision of the paper. QJ conceived the study, supervised the research and revised the manuscript.

\section{Additional material}

\section{Additional file 1}

Mean dN/dS values of 15 protein-coding genes of 6 dermatophyte species. This table lists the mean $d N / d S$ values of 15 protein-coding genes of 6 dermatophyte species.

Click here for file

[http://www.biomedcentral.com/content/supplementary/14712164-10-238-S1.doc]

\section{Additional file 2}

Forty one primers of T. rubrum for LA-PCR test of the other four species and the results. This table includes primers of $\mathrm{T}$. rubrum for $L A-P C R$ test of the other four species and the results.

Click here for file

[http://www.biomedcentral.com/content/supplementary/14712164-10-238-S2.doc]

\section{Acknowledgements}

We are grateful to Ruoyu Li (Research Center for Medical Mycology, Peking University, Beijing, China) for providing dermatophyte strains. Yuan Zhang is thanked for technical assistance in performing phylogenetic analysis. This work was supported under the National High Technology Research and Development Program (Grant No. 2006AA020504) from the Ministry of Science and Technology of China.

\section{References}

I. Weitzman I, Summerbell RC: The dermatophytes. Clin Microbiol $\operatorname{Rev}$ 1995, 8(2):240-259.

2. Wang L, Ma L, Leng W, Liu T, Yu L, Yang J, Yang L, Zhang W, Zhang $\mathrm{Q}$, Dong J, et al.: Analysis of the dermatophyte Trichophyton rubrum expressed sequence tags. BMC Genomics 2006, 7:255.

3. Chermette R, Ferreiro L, J G: Dermatophytoses in animals. Mycopathologia 2008, 166(5-6):385-405.

4. Jousson O, Lechenne B, Bontems O, Capoccia S, Mignon B, Barblan J, Quadroni M, Monod M: Multiplication of an ancestral gene encoding secreted fungalysin preceded species differentiation in the dermatophytes Trichophyton and Microsporum. Microbiology 2004, I50(Pt 2):301-310.

5. Seyfarth F, Ziemer M, Graser Y, Elsner P, Hipler UC: Widespread tinea corporis caused by Trichophyton rubrum with non-typical cultural characteristics - diagnosis via PCR. Mycoses 2007, 50(Suppl 2):26-30.

6. Martin W, Muller M: The hydrogen hypothesis for the first eukaryote. Nature 1998, 392(667I):37-4I.

7. Bullerwell CE, Lang BF: Fungal evolution: the case of the vanishing mitochondrion. Curr Opin Microbiol 2005, 8(4):362-369.

8. Lang BF, Gray MW, Burger G: Mitochondrial genome evolution and the origin of eukaryotes. Annu Rev Genet 1999, 33:35I-397.
9. Gray MW: Evolution of organellar genomes. Curr Opin Genet Dev 1999, 9(6):678-687.

10. Andersson SG, Karlberg O, Canback B, Kurland CG: On the origin of mitochondria: a genomics perspective. Philos Trans $R$ Soc Lond B Biol Sci 2003, 358(1429): 165-177.

II. Burger G, Gray MW, Lang BF: Mitochondrial genomes: anything goes. Trends Genet 2003, I9(I 2):709-716.

12. Tambor JH, Guedes RF, Nobrega MP, Nobrega FG: The complete DNA sequence of the mitochondrial genome of the dermatophyte fungus Epidermophyton floccosum. Curr Genet 2006, 49(5):302-308.

13. Bullerwell CE, Gray MW: Evolution of the mitochondrial genome: protist connections to animals, fungi and plants. Curr Opin Microbiol 2004, 7(5):528-534.

14. Adams KL, Palmer JD: Evolution of mitochondrial gene content: gene loss and transfer to the nucleus. Mol Phylogenet Evol 2003, 29(3):380-395.

15. Dowling DK, Friberg U, Lindell J: Evolutionary implications of non-neutral mitochondrial genetic variation. Trends Ecol Evol 2008, 23(10):546-554.

16. Meiklejohn CD, Montooth KL, Rand DM: Positive and negative selection on the mitochondrial genome. Trends Genet 2007, 23(6):259-263.

17. Blier PU, Dufresne F, Burton RS: Natural selection and the evo-

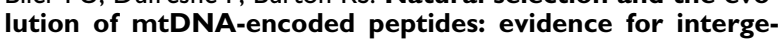
nomic co-adaptation. Trends Genet 200I, 17(7):400-406.

18. Sideris EG, Charalambous SC, Tsolomyty A, Katsaros N: Mutagenesis; carcinogenesis and the metal elements - DNA interaction. Prog Clin Biol Res 1988, 259: 13-25.

19. Clark-Walker GD: Evolution of mitochondrial genomes in fungi. Int Rev Cytol 1992, I 41:89-127.

20. Bertrand H: ROLE OF MITOCHONDRIAL DNA IN THE SENESCENCE AND HYPOVIRULENCE OF FUNGI AND POTENTIAL FOR PLANT DISEASE CONTROL. Annu Rev Phytopathol 2000, 38:397-422.

21. Bullerwell CE, Forget L, Lang BF: Evolution of monoblepharidalean fungi based on complete mitochondrial genome sequences. Nucleic Acids Res 2003, 3 I (6): I6 I4- I 623.

22. Paquin B, Laforest MJ, Forget L, Roewer I, Wang Z, Longcore J, Lang $B F$ : The fungal mitochondrial genome project: evolution of fungal mitochondrial genomes and their gene expression. Curr Genet 1997, 3 I (5):380-395.

23. Yang J, Chen L, Wang L, Zhang W, Liu T, Jin Q: TrED: the Trichophyton rubrum Expression Database. BMC Genomics 2007, 8:250.

24. Anderson JB, Wickens C, Khan M, Cowen LE, Federspiel N, Jones T, Kohn LM: Infrequent genetic exchange and recombination in the mitochondrial genome of Candida albicans. J Bacteriol 200I, I 83(3):865-872.

25. Ghikas DV, Kouvelis VN, Typas MA: The complete mitochondrial genome of the entomopathogenic fungus Metarhizium anisopliae var. anisopliae: gene order and trn gene clusters reveal a common evolutionary course for all Sordariomycetes, while intergenic regions show variation. Arch Microbiol 2006, I85(5):393-40I.

26. Kouvelis VN, Ghikas DV, Typas MA: The analysis of the complete mitochondrial genome of Lecanicillium muscarium (synonym Verticillium lecanii) suggests a minimum common gene organization in mtDNAs of Sordariomycetes: phylogenetic implications. Fungal Genet Biol 2004, 4 I ( I 0):930-940.

27. Pantou MP, Kouvelis VN, Typas MA: The complete mitochondrial genome of Fusarium oxysporum: insights into fungal mitochondrial evolution. Gene 2008, 4 I9(I-2):7-15.

28. Saccone C, Barome PO, D'Erchia AM, D'Errico I, Pesole G, Sbisa E, Tullo A: Molecular strategies in Metazoan genomic evolution. Gene 2002, 300(I-2): 195-20I.

29. Schafer B, Gan L, Perlman PS: Reverse transcriptase and reverse splicing activities encoded by the mobile group II intron cobll of fission yeast mitochondrial DNA. J Mol Biol 2003, 329(2): $191-206$.

30. Breitenberger CA, Browning KS, Alzner-DeWeerd B, RajBhandary UL: RNA processing in Neurospora crassa mitochondria: use of transfer RNA sequences as signals. EMBO J 1985, 4(I): 185-195.

31. Sengupta $S$, Yang $X$, Higgs PG: The mechanisms of codon reassignments in mitochondrial genetic codes. J Mol Evol 2007, 64(6):662-688. 
32. Irimia M, Roy SW: Evolutionary convergence on highly-conserved $3^{\prime}$ intron structures in intron-poor eukaryotes and insights into the ancestral eukaryotic genome. PLoS Genet 2008, 4(8): el $000 \mid 48$.

33. Bievre Cd, Dujon B: Organisation of the mitochondrial genome of Trichophyton rubrumIII DNA sequence analysis of the NADH dehydrogenase subunites $1,2,3,4,5$ and the cytochrome b gene. Curr Genet 1999, 35:30-35.

34. Tehler A, Little DP, Farris JS: The full-length phylogenetic tree from I55I ribosomal sequences of chitinous fungi, Fungi. Mycol Res 2003, I07(Pt 8):901-916

35. Berbee ML, Carmean DA, Winka K: Ribosomal DNA and resolution of branching order among the ascomycota: how many nucleotides are enough? Mol Phylogenet Evol 2000, I 7(3):337-344.

36. Berbee ML: Loculoascomycete origins and evolution of filamentous ascomycete morphology based on I 8S rRNA gene sequence data. Mol Biol Evol I996, I3(3):462-470.

37. Reeb V, Lutzoni F, Roux C: Contribution of RPB2 to multilocus phylogenetic studies of the euascomycetes (Pezizomycotina, Fungi) with special emphasis on the lichen-forming Acarosporaceae and evolution of polyspory. Mol Phylogenet Evol 2004, 32(3): 1036-1060.

38. Keeling PJ, Palmer JD: Parabasalian flagellates are ancient eukaryotes. Nature 2000, 405(6787):635-637.

39. James TY, Kauff F, Schoch CL, Matheny PB, Hofstetter V, Cox CJ, Celio G, Gueidan C, Fraker E, Miadlikowska J, et al.: Reconstructing the early evolution of Fungi using a six-gene phylogeny. Nature 2006, 443(7 I | 3):818-822

40. Graser Y, El Fari M, Vilgalys R, Kuijpers AF, De Hoog GS, Presber W, Tietz $\mathrm{H}$ : Phylogeny and taxonomy of the family Arthrodermataceae (dermatophytes) using sequence analysis of the ribosomal ITS region. Med Mycol |999, 37(2): I05- I |4

41. Graser Y, Scott J, Summerbell R: The new species concept in dermatophytes-a polyphasic approach. Mycopathologia 2008 I 66(5-6):239-256.

42. Juhasz A, Pfeiffer I, Keszthelyi A, Kucsera J, Vagvolgyi C, Hamari Z: Comparative analysis of the complete mitochondrial genomes of Aspergillus niger mtDNA type I a and Aspergillus tubingensis mtDNA type 2b. FEMS Microbiol Lett 2008, 28I(I):5I-57.

43. Woo PC, Zhen H, Cai JJ, Yu J, Lau SK, Wang J, Teng JL, Wong SS, Tse $\mathrm{RH}$, Chen $\mathrm{R}$, et al.: The mitochondrial genome of the thermal dimorphic fungus Penicillium marneffei is more closely related to those of molds than yeasts. FEBS Lett 2003, 555(3):469-477.

44. Taylor TN, Hass $\mathrm{H}$, Kerp $\mathrm{H}$ : The oldest fossil ascomycetes. Nature 1999, 399(6737):648.

45. Harmsen D, Schwinn A, Weig M, Brocker EB, Heesemann J: Phylogeny and dating of some pathogenic keratinophilic fungi using small subunit ribosomal RNA. I Med Vet Mycol 1995 , 33(5):299-303.

46. Hedges SB, Blair JE, Venturi ML, Shoe JL: A molecular timescale of eukaryote evolution and the rise of complex multicellular life. BMC Evol Biol 2004, 4:2.

47. White TC, Oliver BG, Graser Y, Henn MR: Generating and testing molecular hypotheses in the dermatophytes. Eukaryot Cell 2008, 7(8): | 238- I 245

48. Graer Y, Fari ME, Vilgalys R, Kuijpers AFA, Hoog GSD, Presber W, Tietz H-J: Phylogeny and taxonomy of the family Arthrodermataceae (dermatophytes) using sequence analysis of the ribosomal ITS region. Medical Mycology 1999, 37: 105-II4.

49. Leclerc MC, Philippe H, Gueho E: Phylogeny of dermatophytes and dimorphic fungi based on large subunit ribosomal RNA sequence comparisons. J Med Vet Mycol I994, 32(5):331-34I.

50. Summerbell RC, Haugland RA, Li A, Gupta AK: rRNA gene internal transcribed spacer $I$ and 2 sequences of asexual, anthropophilic dermatophytes related to Trichophyton rubrum. J Clin Microbiol 1999, 37( I 2):4005-40 I I.

5I. Makimura K, Tamura Y, Mochizuki T, Hasegawa A, Tajiri Y, Hanazawa R, Uchida K, Saito H, Yamaguchi H: Phylogenetic classification and species identification of dermatophyte strains based on DNA sequences of nuclear ribosomal internal transcribed spacer I regions. J Clin Microbiol I999, 37(4):920-924.

52. Hirai A, Kano R, Nakamura Y, Watanabe S, Hasegawa A: Molecular taxonomy of dermatophytes and related fungi by chitin synthase I (CHSI) gene sequences. Antonie Van Leeuwenhoek 2003, 83(I): II-20.
53. Kano R, Hirai A, Hasegawa A: Chitin synthase I gene of Arthroderma benhamiae isolates in Japan. Mycoses 2002, 45(8):277-28I.

54. Kano R, Nakamura Y, Watanabe S, Tsujimoto H, Hasegawa A: Phylogenetic relation of Epidermophyton floccosum to the species of Microsporum and Trichophyton in chitin synthase I (CHSI) gene sequences. Mycopathologia 1999, I46(3): I I I-II3.

55. Kanbe T, Suzuki Y, Kamiya A, Mochizuki T, Fujihiro M, Kikuchi A: PCR-based identification of common dermatophyte species using primer sets specific for the DNA topoisomerase II genes. J Dermatol Sci 2003, 32(2): |5 |-|6|

56. Pounder JI, Williams S, Hansen D, Healy M, Reece K, Woods GL: Repetitive-sequence-PCR-based DNA fingerprinting using the Diversilab system for identification of commonly encountered dermatophytes. J Clin Microbiol 2005, 43(5):2|4|-2|47.

57. Kanbe T, Suzuki Y, Kamiya A, Mochizuki T, Kawasaki M, Fujihiro M, Kikuchi A: Species-identification of dermatophytes Trichophyton, Microsporum and Epidermophyton by PCR and PCR-RFLP targeting of the DNA topoisomerase II genes. J Dermatol Sci 2003, 33(I):4l-54.

58. Kac G: Molecular approaches to the study of dermatophytes. Med Mycol 2000, 38(5):329-336.

59. Leng W, Liu T, Li R, Yang J, Wei C, Zhang W, Jin Q: Proteomic profile of dormant Trichophyton rubrum conidia. BMC Genomics 2008, 9:303.

60. Bievre $\mathrm{Cd}$, Dujon B: Organisation of the mitochondrial genmone of Trichophyton rubrum DNA sequence analysis of the ND4 gene, the ATPase subunit-6gene, the ribosomal RNA small-subunit gene, the ND6gene, the cox3gene, the ATPase subunit- 8 gene and six tRNA genes that correspond respective to the tyrosine, lysine, glutamine, asparagine, isoleucine and trytophab acceptors. Curr Genet 1995, 28:553-559.

61. Bievre Cd, Dujon B: Mitochondrial DNA sequence analysis of the cytochrome oxidase subunit I and II genes, the ATPase9gene, the NADH dehydrogenase ND4L and ND5 gene complex, and the glutaminy I, methiony I and arginy I tRNA genes from Trichophyton rubrum. Curr Genet 1992, 22:229-234.

62. Cd B, Dujon B: Organisation of the mitochondrial genmone of Trichophyton rubrum DNA sequence analysis of the ND4 gene, the ATPase subunit-6gene, the ribosomal RNA smallsubunit gene, the ND6gene, the cox3gene, the ATPase subunit- 8 gene and six tRNA genes that correspond respective to the tyrosine, lysine, glutamine, asparagine, isoleucine and trytophab acceptors. Curr Genet 1995, 28:553-559.

63. Ewing B, Green P: Base-calling of automated sequencer traces using phred. II. Error probabilities. Genome Res 1998 , 8(3): $186-194$

64. Altschul SF, Madden TL, Schaffer AA, Zhang J, Zhang Z, Miller W, Lipman DJ: Gapped BLAST and PSI-BLAST: a new generation of protein database search programs. Nucleic Acids Res 1997, 25(I 7):3389-3402.

65. Lowe TM, Eddy SR: tRNAscan-SE: a program for improved detection of transfer RNA genes in genomic sequence. Nucleic Acids Res 1997, 25(5):955-964

66. Yang J, Wang J, Yao ZJ, Jin Q, Shen Y, Chen R: GenomeComp: a visualization tool for microbial genome comparison. J Microbiol Methods 2003, 54(3):423-426.

67. Thompson JD, Higgins DG, Gibson TJ: CLUSTAL W: improving the sensitivity of progressive multiple sequence alignment through sequence weighting, position-specific gap penalties and weight matrix choice. Nucleic Acids Res 1994, 22(22):4673-4680.

68. Pond SL, Frost SD: Datamonkey: rapid detection of selective pressure on individual sites of codon alignments. Bioinformatics 2005, 2 I ( I 0):2531-2533.

69. Schmidt HA, Strimmer K, Vingron M, von Haeseler A: TREE-PUZZLE: maximum likelihood phylogenetic analysis using quartets and parallel computing. Bioinformatics 2002, I 8(3):502-504

70. Abascal F, Zardoya R, Posada D: ProtTest: selection of best-fit models of protein evolution. Bioinformatics 2005 , 2 I (9):2 104-2105.

7I. Tamura K, Dudley J, Nei M, Kumar S: MEGA4: Molecular Evolutionary Genetics Analysis (MEGA) software version 4.0. $\mathrm{Mo}$ Biol Evol 2007, 24(8): I596-I599. 